\title{
Universiteit
}

Leiden

The Netherlands

\section{Rod-Climbing Effect in Newtonian Fluids}

Bonn, D.; Kobylko, M.; Bohn, S.; Meunier, J.; Morozov, A.; Saarloos, W. van

\section{Citation}

Bonn, D., Kobylko, M., Bohn, S., Meunier, J., Morozov, A., \& Saarloos, W. van. (2004). RodClimbing Effect in Newtonian Fluids. Physical Review Letters, 93(21), 214503.

doi:10.1103/PhysRevLett.93.214503

Version: $\quad$ Not Applicable (or Unknown)

License: $\quad$ Leiden University Non-exclusive license

Downloaded from: https://hdl.handle.net/1887/66547

Note: To cite this publication please use the final published version (if applicable). 


\title{
Rod-Climbing Effect in Newtonian Fluids
}

\author{
Daniel Bonn, ${ }^{1,2, *}$ Mathias Kobylko, ${ }^{1}$ Steffen Bohn, ${ }^{1}$ Jacques Meunier, ${ }^{1}$ Alexander Morozov, ${ }^{3}$ and Wim van Saarloos ${ }^{3}$ \\ ${ }^{1}$ Laboratoire de Physique Statistique, Ecole Normale Supérieure, 24 Rue Lhomond, 75231 Paris cedex 05, France \\ ${ }^{2}$ van der Waals--Zeeman Institute, University of Amsterdam, Valckenierstraat 65, 1018 XE Amsterdam, The Netherlands \\ ${ }^{3}$ Instituut-Lorentz, University of Leiden, Postbus 9506, 2300 RA Leiden, The Netherlands
}

(Received 14 April 2004; published 18 November 2004)

\begin{abstract}
When a rotating rod is brought into a polymer melt or concentrated polymer solution, the meniscus climbs the rod. This spectacular rod climbing is due to the normal stresses present in the polymer fluid and is thus a purely non-Newtonian effect. A similar rod climbing of an interface between two fluids has therefore been taken as a signature that one of the fluids exhibits normal stress effects. We show here, however, that the effect can occur with simple Newtonian fluids: it occurs when a Taylor-Couette instability happens in the less viscous of the two liquids but not in the more viscous one.
\end{abstract}

DOI: $10.1103 /$ PhysRevLett.93.214503

PACS numbers: 47.20.-k, 83.60.Hc

One of the textbook illustrations [1] of the remarkable flow behavior of polymer melts and solutions is the socalled rod-climbing or Weissenberg effect: when a rotating rod is immersed in a normal Newtonian fluid, the meniscus descends due to the centrifugal forces. However, when a rotating rod is immersed in a concentrated polymer melt or solution, the meniscus climbs the rod. This effect is due to the normal stresses that the shear induces in the polymeric fluid: the shear stretches and orients the polymers. The difference in the normal components of the stress tensor that this anisotropy induces is such that the fluid is pulled inwards and climbs the rod [1].

In this Letter, we demonstrate that when a rotating rod is immersed into a beaker with two immiscible Newtonian fluids, surprisingly the interface between the two fluids can also climb the rod, provided the less viscous liquid is also the heavier one. However, if the viscosity contrast between the two fluids is inverted, the meniscus is observed to descend. In addition, we find that there is a clear threshold for the onset of rod climbing. All these observations can be understood in terms of the occurrence of a Taylor-Couette (TC) instability $[2,3]$ in the less viscous of the two fluids.

Thus, although the effect is visually akin to the spectacular rod climbing of polymeric fluids, it should not be mistaken as a signature of normal stress effects in one of the two fluids. In fact, our study was partly motivated by the occurrence of rod climbing in a two-phase surfactant system of liposomes. For this system, the rod climbing was taken as evidence of unusual normal stress effects due to an entangled tubular network in one of the two coexisting phases [4]. However, when performing the rheology of this system, we did not find any measurable normal stress effect for shear rates between $10^{-2}$ and $1000 \mathrm{~s}^{-1}$ (data not shown), our detection level being on the order of $5 \mathrm{~Pa}$. The fact that our own rheological measurements did not, in fact, exhibit any nonNewtonian behavior led us to investigate the effect in detail.
We therefore performed the rotating rod experiment on two perfectly Newtonian fluids. A typical experiment consists of simply lowering a rod into a beaker filled with water (of viscosity $\eta=1 \mathrm{mPas}$ ) and a viscous silicon oil ( $\eta=100 \mathrm{mPas})$. When the rod is made to rotate, the meniscus starts to climb the rod [Fig. 1(a)]. If the viscosity contrast is inverted using viscous glucose syrup $(\eta=100 \mathrm{mPas})$ as the lower phase and a light silicon oil $(\eta=10 \mathrm{mPas})$ as the upper phase, the effect inverses: the meniscus descends into the lower phase [Fig. 1(b)]. This symmetry between ascend and descend is consistent with simple estimates that show that centrifugal forces are negligible.

In the following, we focus on the rod climbing, i.e., with the less viscous fluid as the heavier phase. For the climbing, we found that one has to exceed a certain rotation rate $\Omega_{c}(\approx 5 \mathrm{~Hz}$ if the lower fluid is water) in order for the rod climbing to occur: the height $h$ is zero below $\Omega_{c}$, and increases roughly linearly with frequency above the critical rotation rate [Fig. 2(a)]. Experiments with a cylinder that is much longer $(30 \mathrm{~cm})$ show the same results, showing that the effect is not due to the finite depth of the fluid. Upon varying the viscosity of the two phases independently, it became evident that the critical rotation frequency $\Omega_{c}$ depends on the viscosity of the less viscous fluid but not notably on that of the more viscous fluid. We find that the onset of rod climbing increases linearly with increasing viscosity of the lower fluid [Fig. 2(b)].

In order to understand these observations, note that a beaker with a rotating rod is, in fact, a version of a TaylorCouette cell, in which a liquid is confined between two concentric cylinders [2,3]. When the inner cylinder of a Taylor-Couette cell is made to rotate, the basic laminar (azimuthal) flow becomes unstable at a critical rotation rate $\Omega^{\mathrm{TC}}$ whose value is determined by the Taylor number. The critical Taylor number can be calculated [2]; if one is not in the limit of small gap, the expression becomes rather complicated, but can easily be evaluated numeri- 

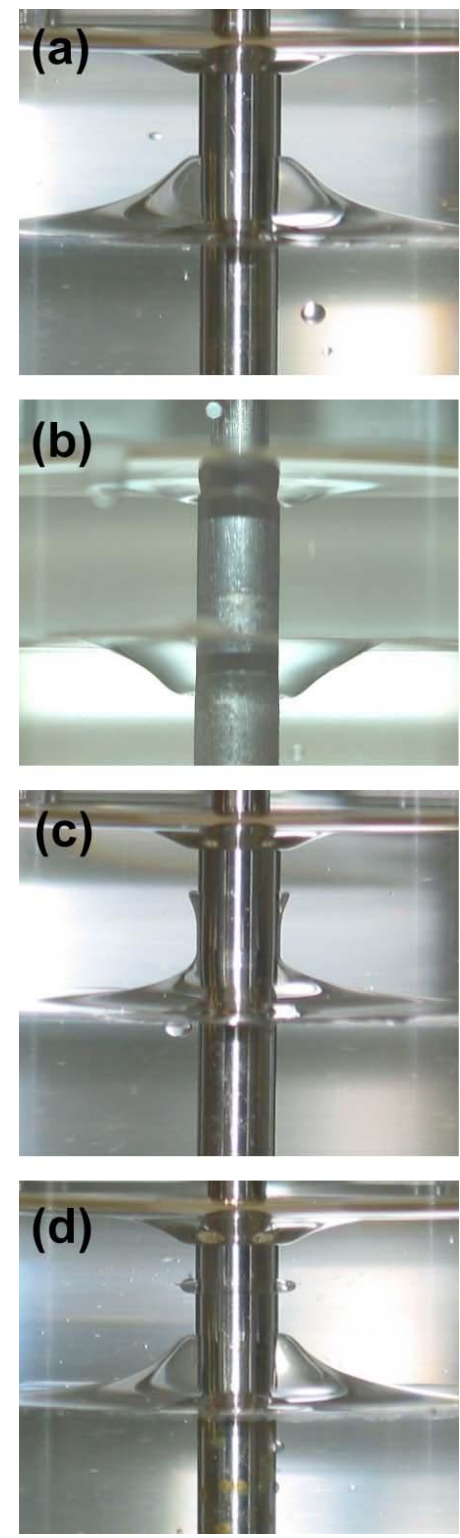

FIG. 1 (color online). Pictures of the interface deformations taken at the interface of the two liquids. (a) Newtonian rod climbing: the lower fluid is water, the upper fluid a $500 \mathrm{mPas}$ silicon oil ( $f=15 \mathrm{~Hz}$ ). (b) If the viscosity contrast is inversed, the interface descends; the lower fluid is glucose syrup (viscosity $300 \mathrm{mPas}$ ) and the upper fluid a light silicon oil (viscosity $10 \mathrm{mPas})(f=20 \mathrm{~Hz})$. (c),(d) Destabilization of the interface for the Newtonian rod climbing; the lower fluid is water, the upper fluid a $500 \mathrm{mPas}$ silicon oil $(f=28$ and $32 \mathrm{~Hz}$, respectively). The setup is a cylindrical glass cell with an inner diameter of $55 \mathrm{~mm}$ and a height of $90 \mathrm{~mm}$. The cell is filled with the heavier liquid, after which the lighter liquid is deposited gently on top of the first liquid. A stainless steel rod of $5 \mathrm{~mm}$ diameter and $100 \mathrm{~mm}$ height is then placed in the center and made to rotate.

cally; it depends on the ratio of the radii $R_{1}$ and $R_{2}$ of the inner and outer cylinders. For us, the relevant feature is that $\Omega^{\mathrm{TC}}$ is proportional to the kinematic viscosity $\nu$ of the fluid, with a prefactor that can be evaluated explicitly
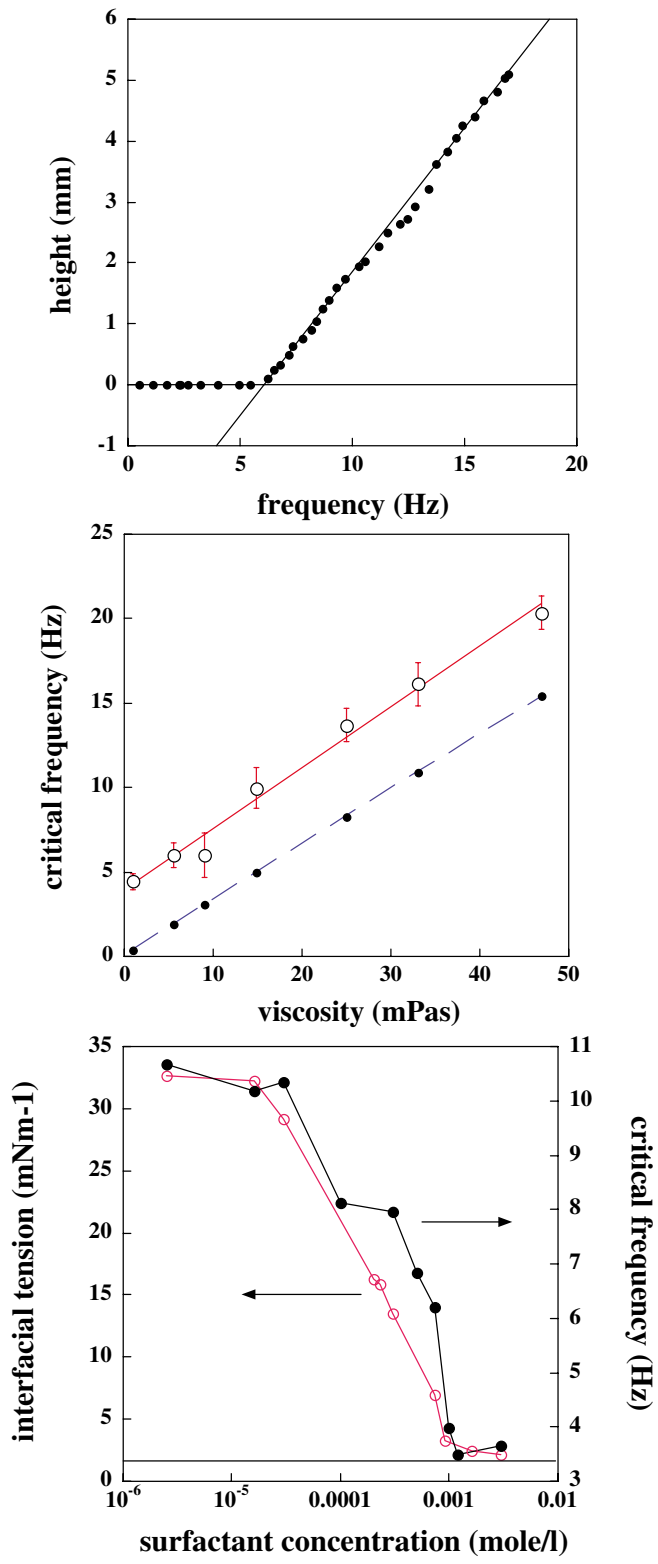

FIG. 2 (color online). (a) Newtonian rod climbing: height of the meniscus as a function of the rotation frequency of the rod. Lower fluid: water; upper fluid: silicon oil, viscosity $300 \mathrm{mPas}$. (b) Critical frequency determined from the intersect of the low and high frequency branches as shown in (a), as a function of the viscosity of the lower fluid. Viscosity of the upper fluid is 300 mPas. Filled symbols are the calculated critical frequencies for the onset of the Taylor-Couette instability in our setup. (c) Interfacial tension and critical frequency as a function of the surfactant concentration; viscosity of the lower fluid is $10 \mathrm{mPas}$. The drawn line gives the theoretical result for the critical frequency without surface tension.

for our cell geometry. Furthermore, from the earlier work it is known that just beyond threshold, at dimensionless control parameters $\varepsilon=\left(\Omega-\Omega^{\mathrm{TC}}\right) / \Omega^{\mathrm{TC}}<\varepsilon_{c} \approx$ 0.3 , a periodic vortex flow state appears and for $\varepsilon>\varepsilon_{c}$ a temporally modulated "wavy vortex flow" state occurs [5-8]. 
In our experiments, the flow state that occurs for small $\Omega$ is the same in the two fluids, since the azimuthal flow field below the TC threshold is independent of the fluid viscosity [2]. However, when $\Omega$ becomes sufficiently large, the bulk flow in the lower viscosity fluid becomes unstable to Taylor vortex flow at a critical rotation rate $\Omega^{\mathrm{TC}}$, while the flow in the more viscous fluid remains essentially in the viscometric regime; the recirculations in the lower liquid are then at the origin of the rod climbing [9]. This is borne out by a direct measurement of the flow field in the lower fluid using PIV (particle image velocimetry). An example of the flow pattern is shown in Fig. 3, which clearly shows the recirculations that occur beyond the onset of the Taylor-Couette instability.

Changing the viscosity of the lower fluid should consequently change the onset of rod climbing, which is indeed what we observe. The critical frequency $\Omega_{c}$ of the rod climbing (obtained by extrapolating our data for height as a function of frequency to zero height) increases linearly with the viscosity of the lower fluid, as one would expect if the Taylor-Couette instability is at the origin of the climbing. We can compare the critical frequencies with those for the onset of the Taylor-Couette instability $[2,8]$. Since we are not in the limit of the small aspect ratio, the onset Taylor number has to be calculated numerically. Comparing the calculation with the experimental results [Fig. 2(b)], we observe that the two lines are parallel, but do not have the same intercept: the rod climbing becomes visible only well after the TaylorCouette instability has set in.

These observations can be understood in a more quantitative way as follows. The instability being supercritical, the amplitude of the velocity of the Taylor vortex flow will grow as $\sqrt{\varepsilon}$. Measurements in the wavy vortex flow regime [6] have shown that this $\sqrt{\varepsilon}$ scaling even extends far into this regime. A realistic calculation of the effect of the vortex velocity field on the interface is difficult, but we can understand the flow-induced "rod climbing" as follows. The pressure $\Delta p_{\text {grav }}$ needed to displace the meniscus a distance $h$ upwards is $\Delta \rho g h$, where $\Delta \rho$ is the density difference between the two fluids and $g$ the gravitational acceleration. For our water-silicon oil system, with a $3 \%$ density difference, the pressure needed to displace the fluid by $1 \mathrm{~mm}$ is about $0.3 \mathrm{~Pa}$. The other two terms with which $\Delta p_{\text {grav }}$ has to balance at the interface are the capillary pressure $\Delta p_{\text {cap }}=\gamma / R$, where $R$ is a typical radius of curvature of the interface, and the effective pressure $\Delta p_{\text {flow }}$ induced by the flow. Let us start with the case of negligible $\Delta p_{\text {cap }}$. The flow-induced pressure $\Delta p_{\text {flow }}$ is estimated from Bernouilli's equation to be of order $\Delta p_{\text {flow }} \approx \rho v^{2} / 2$, where $\rho$ is the density of the fluid where the instability occurs, and where $v$ is the typical amplitude of the velocity [9]. Clearly, $\Delta p_{\text {flow }}$ is linear in $\varepsilon$, since as we saw above $v \sim \sqrt{\varepsilon}$, leading $h \approx$ $\rho v^{2} /(2 \Delta \rho g)$. From [6,8] it follows that $v \approx 10(\eta / \rho d) \sqrt{\varepsilon}$, with $d=R_{2}-R_{1}$ the gap between the cylinders, and thus $h \approx 100 \rho \eta^{2} /\left(2 \Delta \rho g \rho^{2} d^{2}\right) \varepsilon$. This shows that the $\operatorname{rod}$ climbing should set in above the Taylor instability, and that the effect is linear $\varepsilon$, hence linear in $\Omega-\Omega^{\mathrm{TC}}$, as is found in the experiments. However, if we put in the numbers, the prefactor is found to be too small by at least 2 orders of magnitude to give a $h$ of a few millimeters for $\varepsilon \approx 1$.

In the above argument we ignored the capillary pressure $\Delta p_{\text {cap }}$ which tends to keep the meniscus flat; for our water-oil interface and for a typical radius of curvature $R$ of the order of a cm, $\Delta p_{\text {cap }} \approx 35 \mathrm{~Pa}$, much larger $\Delta p_{\text {grav }}$. Therefore, $\Delta p_{\text {flow }}$ is much too small to overcome the capillary term, and the surface will not rise visibly - in effect, the rod climbing will become significant only further above threshold, once the velocity has grown large enough. When the capillary term is reduced, the onset of the rise should shift towards the onset of the Taylor vortex flow.

To test this, we performed measurements in which the capillary pressure term was reduced by adding a surfactant (L77 trisiloxane) that allows one to vary the oilwater interfacial tension continuously by more than an order of magnitude [Fig. 2(c)]. We find that the onset frequency is strongly affected by the lowering of the tension. In Fig. 2(c) we see that when the interfacial tension is reduced, $\Omega_{c}$ drops. These results confirm that the Taylor-Couette instability is at the origin of the climbing: when the interfacial tension is reduced sufficiently, $\Omega_{c}$ and $\Omega^{\mathrm{TC}}$ coincide within the experimental accuracy.

The last problem we need to address is therefore the amplitude of the effect. The above estimates show that the bulk flow is too small to explain the effect quantitatively. The example of the flow pattern shown in Fig. 3 shows that already near $\Omega_{c}$ the flow is significantly enhanced

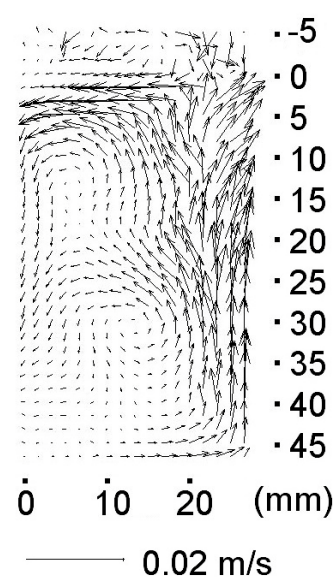

FIG. 3. Velocity field in radial-height plane the lower fluid measured by particle imaging velocimetry. Only half of the cell is shown: the left boundary of the graph corresponds to the rotating rod, whereas the right boundary is the wall of the cylinder. Lower fluid is water, and the rotation rate $10 \mathrm{~Hz}$. 
near the meniscus - the order of magnitude of the flow amplitude away from the meniscus is in agreement with experimental and theoretical values, but near the meniscus the flow amplitude is enhanced by up to a factor of 10 . When we use the order of magnitude of the measured flow amplitude in the above estimate for the height of the rod climbing, we do get values of order $\mathrm{mm}$ indeed. As a final point, we note that one cannot rule out that the perturbation of the flow field near the meniscus makes the behavior weakly subcritical. Our visual observations presently do not exclude a weak subcritical behavior for height elevations of less than about $0.5 \mathrm{~mm}$.

In summary, rod climbing may occur when one looks at the interface between two immiscible Newtonian fluids, provided the heavier fluid has the lower viscosity. If surface tension is negligible, the critical rotation rate for the onset of climbing coincides with that of the TaylorCouette instability. Our observations reconcile the paradoxical "inverse Weissenberg effect" reported at the interface between two coexisting phases in a surfactant solution [4] with our finding that the viscosity and normal stresses of these solutions show Newtonian behavior: the climbing is not a non-Newtonian effect but the Newtonian flow-induced climbing effect studied here.

One potential application of the Newtonian rodclimbing effect is in emulsification: when the climbing of the meniscus becomes comparable to the height of the upper phase, a series of secondary instabilities occurs [Figs. 1(c) and 1(d)] leading to the breakup of the interface near the free surface. Through this breakup, a spray of small droplets of the dense fluid forms in the upper phase. The onset frequency $(30 \mathrm{~Hz}$ for water) is found not to depend significantly on the fluid height of either fluid, nor on the viscosity of the more viscous fluid. The efficiency of this emulsification process is so large that at every time that this happened during the experiments, we had to throw away the upper fluid, since in a few seconds it had become completely opaque. Whether or not such instabilities are due to the stratified flow near the inter- face [2], induced by the Taylor-Couette instability of the low-viscosity fluid, is at present unclear to us.

We thank P. Petitjeans and O. Cadot, T. Bohr, and J. Bush. LPS de l'ENS is UMR 8550 of the CNRS, associated with the universities P6 and P7.

*Electronic address: bonn@lps.ens.fr

[1] R. B. Bird, R. C. Armstrong, and O. Hassager, Dynamics of Polymeric Liquids, Fluid Mechanics Vol. 1 (Wiley, New York, 1987).

[2] S. Chandrasekhar, Hydrodynamic and Hydromagnetic Stability (Dover, New York, 1981).

[3] R. C. DiPrima and H. L. Swinney, in Hydrodynamics and the Transition to Turbulence, edited by H. L. Swinney and J. P. Gollub (Springer, Berlin, 1985).

[4] S. Chiruvolu, H. E. Warriner, E. Naranjo, S. H. J. Idziak, J. O. Rædler, R. J. Plano, J. A. Zasadzinski, and C. R. Safinya, Science 266, 1222 (1994).

[5] C. D. Andereck, S.S. Liu, and H. L. Swinney, J. Fluid Mech. 164, 155 (1986).

[6] S. T. Wereley and R. M. Lueptow, J. Fluid Mech. 364, 59 (1998).

[7] References [5,6] report the transition to the wavy vortex flow state for cells with a gap ratio of about 0.85 ; ours is 0.1. Comparison of Ref. [6] and S. T. Wereley and R. M. Lueptow, J. Fluid Mech. 364, 59 (1998), indicates that the velocity amplitude does not change significantly when the transition from the Taylor vortex flow to the wavy vortex flow occurs, so our interpretation of the experiments does not rely on where the transition happens precisely in our system.

[8] A. Recktenwald, M. Lücke, and H.W. Müller, Phys. Rev. E 48, 4444 (1993).

[9] This explains earlier observations of transient deformations in start-up Couette flow [see A.S. Berman, J. Bradford, and T. S. Lundgren, J. Fluid Mech. 84, 411 (1978)].Heating induced instabilities [J. M. White and S. J. Muller, Phys. Rev. Lett. 84, 5130 (2000)] can be ruled out here because of the small viscosities of our liquids. 